\title{
Predictions of biodiversity are improved by integrating trait-based competition with abiotic filtering
}

\author{
Loïc Chalmandrier ${ }^{1,2,3}$ ( ) Daniel B. Stouffer ${ }^{2} \odot$ | Adam S. T. Purcell ${ }^{4}$ |

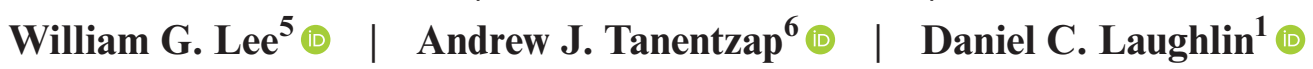

${ }^{1}$ Department of Botany, University of Wyoming, Laramie, Wyoming, USA

${ }^{2}$ Centre for Integrative Ecology, School of Biological Sciences, University of

Canterbury, Christchurch, New Zealand

${ }^{3}$ Theoretical Ecology, Faculty of Biology and Pre-Clinical Medicine, University of

Regensburg, Regensburg, Germany

${ }^{4}$ Titoki Landcare, Hamilton, New Zealand

${ }^{5}$ Landcare Research, Dunedin, New

Zealand

${ }^{6}$ Ecosystems and Global Change Group, University of Cambridge, Cambridge, UK

\section{Correspondence}

Loï Chalmandrier, Department of Botany, University of Wyoming, Laramie, WY,

USA.

Email: loic.chalmandrier@biologie.uni-

regensburg.de

Funding information

University of Canterbury; University of Waikato; H2020 Marie Skłodowska-Curie Actions, Grant/Award Number: 840946;

University of Wyoming; Manaaki Whenua

- Landcare Research; New Zealand

Government, Grant/Award Number: 16-

UOC-008 and RDF-13-UOC-003

Editor: Akira Mori

\begin{abstract}
All organisms must simultaneously tolerate the environment and access limiting resources if they are to persist. Approaches to understanding abiotic filtering and competitive interactions have generally been developed independently. Consequently, integrating those factors to predict species abundances and community structure remains an unresolved challenge. We introduce a new synthetic framework that models both abiotic filtering and competition by using functional traits. First, our framework estimates species carrying capacities along abiotic gradients. Second, it estimates pairwise competitive interactions as a function of species trait differences. Applied to the study of a complex wetland community, our combined approach more than doubles the explained variance of species abundances compared to a model of abiotic tolerances alone. Trait-based integration of competitive interactions and abiotic filtering improves our ability to predict species abundances, bringing us closer to more accurate predictions of biodiversity structure in a changing world.
\end{abstract}

\section{K E Y W O R D S}

abiotic filtering, community modelling, competition, functional trait, plant ecology, species

abundances, wetland

\section{INTRODUCTION}

Predicting species abundances is a major focus of community ecology (McGill et al., 2006). In recent decades, trait-based ecology has proposed that species morphological, physiological or phenological features determine how abiotic filtering and species interactions affect local community structure (Kraft, Adler, et al., 2015; Violle et al., 2007). However, trait-based analyses of communities often focus on functional diversity (Chalmandrier et al., 2017; Spasojevic et al., 2014) and few explicitly model species abundances (Zakharova et al., 2019).

Trait-based models of abiotic filtering that statistically estimate species abundances assume that there are optimum trait values within a given environment. Species able to attain these trait values will be more likely occur in that environment (Laughlin et al., 2012; Shipley, 2010). In parallel, theoretical models of species interactions have a long and storied history in ecology (Chesson, 2000; Lotka, 1926) and have been used to understand the foundational conditions for coexistence among competing species. For species

This is an open access article under the terms of the Creative Commons Attribution License, which permits use, distribution and reproduction in any medium, provided the original work is properly cited.

(C) 2022 The Authors. Ecology Letters published by John Wiley \& Sons Ltd. 
to coexist stably, niche differences among species must be greater than differences in competitive ability (Adler et al., 2007; Chesson, 2000) and recent work suggests that those differences can be linked to functional traits (Kraft, Godoy, et al., 2015). Advances in these two classes of models have largely proceeded independently (Kraft, Adler, et al., 2015), and hence, we still lack well-established and broadly testable approaches that capture both perspectives (but see D'Amen et al., 2015; Ovaskainen et al., 2017).

Three primary obstacles have prevented the mathematical integration of models of abiotic filtering and models of species interactions. First, they lack a common numerical currency through which they could be linked. Trait-based models of abiotic filtering yield probabilities that a species occurs in an environment given its traits, whereas models of species interactions describe the dynamics of populations over time or equilibrium abundances given growth rates, carrying capacities and pairwise interaction coefficients (Chesson, 2000; Lotka, 1926). Second, the complexity of estimating pairwise interactions increase exponentially with the number of species in the community, and there has been no obvious method for estimating interaction coefficients without implementing laborious competition experiments (Kraft, Godoy, et al., 2015; Narwani et al., 2013). Finally, there have been no adequate tools to model classical community ecology sampling schemes. For instance, plant abundance is often visually assessed through percent cover classes that do not necessarily fit well with existing statistical frameworks. Recently, authors have formalised the use of beta distributions to adequately model these sampling schemes (Damgaard \& Irvine, 2019), but they have yet to be implemented in biodiversity modelling.

Here, we present a new synthetic framework that overcomes these three obstacles. This framework, which we call Banquo (Shipley, 2010), integrates Traitspace, a traitbased model of abiotic filtering (Laughlin et al., 2012), with a Lotka-Volterra competition model. First, we assume that the probability that a species occurs in an environment given its traits determines its local carrying capacity, that is, the maximum population size that a species can reach locally in the absence of interspecific competition (MacArthur \& Levins, 1967). Second, we assume pairwise interaction coefficients can be represented as a function of observed trait differences between species (Kraft, Godoy, et al., 2015), thereby substantially reducing the number of parameters needed to estimate pairwise interaction coefficients (Chalmandrier et al., 2021). Drawing inspiration from coexistence theory (Adler et al., 2007; Chesson, 2000), the parameterisation of this function allows for competitive outcomes to be affected by a mixture of niche partitioning (i.e. strong competitive interference among functionally similar species) and competitive hierarchies (i.e. species have strong competitive impacts on species with inferior trait values). Third, we use the recent methodological developments of Irvine et al. (2019) to link the output of our framework to observed plant species covers estimated through cover classes.
We illustrate our framework by modelling plant species covers along a flooding gradient in an ephemeral wetland (Purcell et al., 2019). After presenting our framework, we calibrated 18 assembly models that include abiotic filtering and/or biotic filtering tested on different sets of functional traits. Then, we compared the statistical performance of these 18 assembly models. Finally, we analysed how the parameterisation and output of the calibrated models inform our knowledge about the assembly of wetland plant communities.

\section{METHODS}

\section{The framework}

\section{Step 1-Estimating species carrying capacities along environmental gradients}

We started with the Traitspace framework to model species' probabilities of occurrence along environmental gradients (Laughlin et al., 2012). Traitspace characterises the size and shape of the environmental filter based on a multivariate linear model with a vector of individual plant traits $(T)$ as the response and a vector of environmental gradients $(E)$ as the predictors, that is, the function $T=f(E)$. Traitspace uses this linear model to estimate the conditional distributions of traits $T$ given the environmental conditions in site $k\left(P\left(T \mid E_{k}\right)\right)$. Second, it uses the intraspecific trait distribution of each species across sites, that is, the conditional distributions of traits given species identity $\left(P\left(T \mid S_{i}\right)\right)$. The posterior distribution of species presence $S_{i k}$ of species $i$ in site $k$ is conditioned on both the trait state $T$ and the environmental conditions $E_{k} . P\left(S_{i k} \mid T, E_{k}\right)$ is computed using Bayes theorem:

$$
P\left(S_{i k} \mid T, E_{k}\right)=\frac{P\left(T \mid S_{i .}\right) P\left(S_{i k}\right)}{\sum_{i} P\left(T \mid S_{i}\right) P\left(S_{i k}\right)} .
$$

The posterior is computed by integrating with respect to traits to obtain the probability of occurrence of a species given the environmental conditions:

$$
P\left(S_{i k} \mid E_{k}\right)=\int P\left(S_{i k} \mid T, E_{k}\right) d T
$$

In practice, we use Monte Carlo integration to estimate the average probability of the presence of each species in each site by randomly sampling 500 trait values per site based on the estimated trait-environment relationship $(T=f(E))$ and then average the probability distribution for each site and each species. In the end, Traitspace produces a site-by-species probability matrix.

Extending the original Traitspace framework, we then assumed that the carrying capacity (in percent cover) $K_{i k}$ of 
species $i$ in a site $k$ can be estimated from its probability of presence in that site using an increasing $\log -\log$ function:

$$
K_{i k}=a\left(\frac{P\left(S_{i k} \mid E_{k}\right)}{\max _{i, k}\left(P\left(S_{i k} \mid E_{k}\right)\right)}\right)^{b} .
$$

With $a \in[0,1]$, and $b$ is positive real number $\left(b \in \mathbb{R}^{+}\right)$.

We standardised the probability value $P\left(S_{i k} \mid E_{j}\right)$ by the maximum value across all species $i$ and all sites $k$ to ensure that carrying capacities $K_{i k}$ are all set between 0 and 1 (as a percent cover variable). This implies that the parameter $a$ then represents the maximum possible value of $K_{i k}$.

\section{Step 2-Estimating competitive interactions}

\section{Formulation of the interaction matrix}

Here, we assume that the interaction coefficient $\alpha_{i j}$ that measures the competitive impact of species $j$ on species $i$ is a function of trait differences. We test a formulation of $\alpha_{i j}$ as a function of the empirical trait difference between species $i$ and species $j\left(\Delta t_{i j}=t_{i}-t_{j}\right)$ :

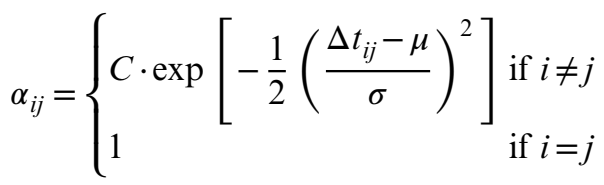

with $C \in \mathbb{R}^{+}, \mu \in \mathbb{R}, \sigma \in \mathbb{R}^{+}$.

Interspecific coefficients follow a Gaussian function of trait differences where $\mu$ is the peak position of the Gaussian, $\sigma$ is its width, and $C$ controls the amplitude of interspecific coefficients. Species intraspecific coefficients were fixed to 1 . For small values of $C$, the matrix of interaction coefficients can be approximated by the identity matrix $(\boldsymbol{\alpha}=\boldsymbol{I})$ and estimated species covers simplify to species carrying capacities. For large values of $\sigma(\sigma \rightarrow \infty)$, interspecific coefficients are all equal to $C$ and represent a situation where interspecific interactions among species are constant and do not depend on species traits.

The formulation of Equation 4 can be directly related to competitive hierarchies and niche partitioning among species (Chesson, 2020). The value of the parameter $\mu$ determines if the studied trait relates more to niche partitioning, hierarchical competition or a mixture of the (a)

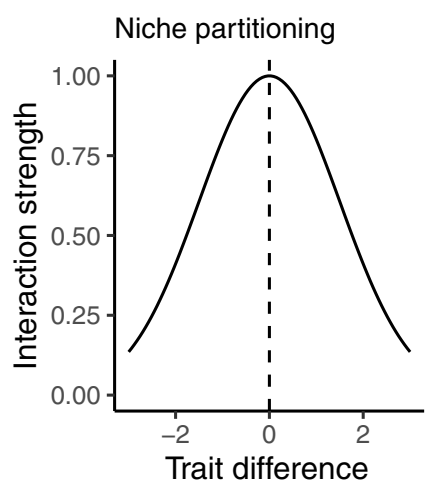

(d) Niche partitioning

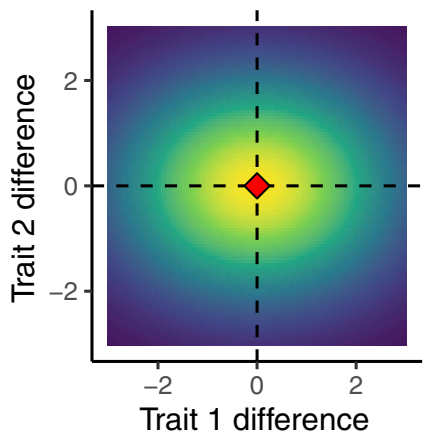

(b)

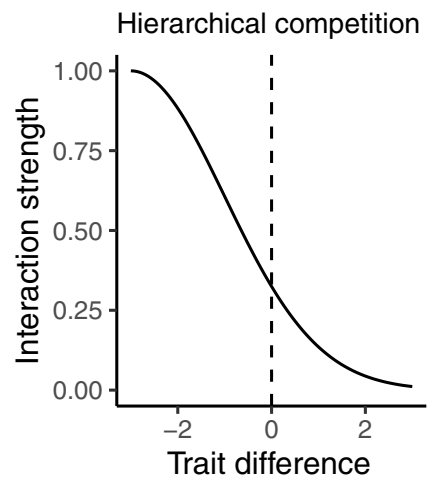

(e) Niche partitioning and hierarchical competition

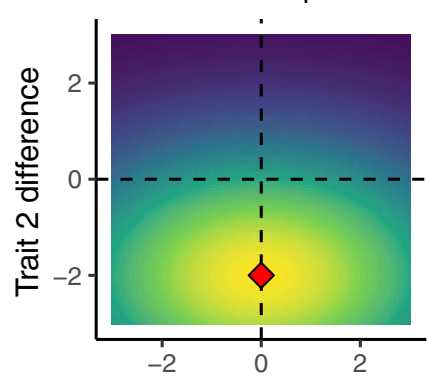

Trait 1 difference (c)
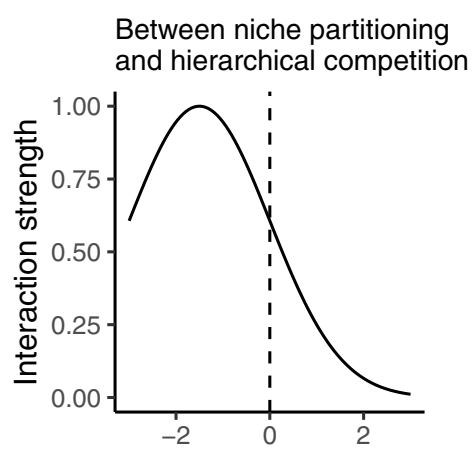

Trait difference

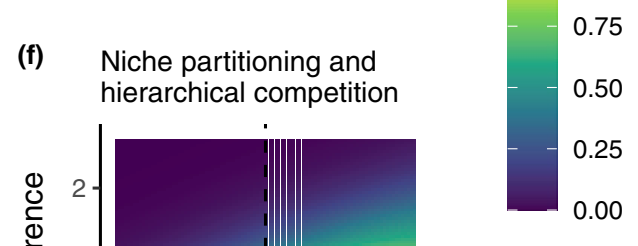

0.00

F I G U R E 1 Illustration of our modelling of interspecific pairwise interaction coefficients. They are defined as a function of standardised trait differences that depend on peak position $(\mu)$ and width $(\sigma)$ parameters (see Methods). (a) Niche partitioning $(C=1, \mu=0, \sigma=1.5)$. (b) Hierarchical competition $(C=1, \mu=-3, \sigma=2)$. (c) Intermediate situation between niche partitioning and hierarchical competition $(C=1, \mu=1.5$, $\sigma=1.5)$. (d) Niche partitioning along the two trait dimensions $\left(C=1, \mu_{1}=\mu_{2}=0, \sigma_{1}=2, \sigma_{2}=1.5, \rho_{21}=0\right)$. (e) Niche partitioning along trait 1 differences and hierarchical along trait 2 differences $\left(C=1, \mu_{1}=0, \mu_{2}=-2, \sigma_{1}=3, \sigma_{2}=2, \rho_{21}=0\right)$. (f) Niche partitioning along trait 1 differences and hierarchical along trait 2 differences with an interaction among both dimensions $\left(C=1, \mu_{1}=0, \mu_{2}=-1, \sigma_{1}=4, \sigma_{2}=2, \rho_{21}=0.8\right)$. Orange diamonds in panels $(\mathrm{d}-\mathrm{f})$ indicate the position of the largest pairwise interaction coefficient 
two. Specifically, for $\mu$ close to 0 , pairwise interaction coefficients are high for small trait differences and low for large trait differences, indicating a predominance of niche partitioning among species (Figure 1a). For a large and negative value of $\mu$, the left hand part of the bell-shaped curve falls outside of the range of observed trait differences. Thus, the curve approaches a monotonous function that indicates a predominance of competitive hierarchy: Species with a large trait value are competitively superior over species with small trait values (Figure 1b). Conversely, a large and negative value of $\mu$ indicates that species with a small trait value are competitively superior over species with large trait values. Less extreme values of $\mu$ indicate an intermediate situation (Figure 1c): Niche partitioning is predominant among species with large trait differences, but among species with small trait differences, competition is not symmetric (as in a case of 'pure' niche partitioning) and hierarchical competition is the predominant coexistence process. We further show in the supplementary information how these features relate to the classical two-species coexistence framework (Chesson, 2020).

Finally, our formulation can be extended to multiple trait dimensions using a multivariate Gaussian function. Interaction coefficients are then formulated as a function of the vector of trait differences between species $i$ and $j, \boldsymbol{\Delta} \boldsymbol{t}_{i j}=\left[t_{1, i}-t_{1, j}, t_{2, i}-t_{2, j}\right]$.

$$
\alpha_{i j}= \begin{cases}C \cdot \exp \left[-\frac{1}{2}\left(\Delta t_{i j}-\boldsymbol{\mu}\right)^{T} \boldsymbol{\Sigma}^{-1}\left(\Delta \boldsymbol{t}_{i j}-\boldsymbol{\mu}\right)\right] & \text { if } i \neq j \\ 1 & \text { if } i=j\end{cases}
$$

with $C \in \mathbb{R}^{+}, \boldsymbol{\mu}=\left[\mu_{1}, \mu_{2}, \ldots\right] \in \mathbb{R}^{N}$. $\boldsymbol{\Sigma}$ is a covariance matrix (symmetric and positive semidefinite matrix of dimension $N \times N)$.

This equation describes an $N$-dimensional Gaussian function of $\Delta \boldsymbol{t}_{i j}$ of peak position $\left(\mu_{1}, \mu_{2}, \ldots\right)$ across the successive trait dimensions. $\boldsymbol{\Sigma}$ is a covariance matrix obtained from the width parameters $\sigma_{1}, \sigma_{2}, \ldots$ and parameters $\rho_{l m}(1 \leq m<l \leq N)$. If $m=1, \rho_{l m}$ is the correlation coefficient between trait $l$ and trait $m$. If $t>1, \rho_{l m}$ is the partial correlation coefficient between trait $l$ and trait $m$ after removing the effect of trait 1 to $m-1$. The parameters $\rho_{l m}$ control the Cholesky factorisation of the correlation matrix among trait dimensions (more details in Forrester and Zhang 2020).

Properties and interpretations of the parameters are similar to their unidimensional counterparts. For instance, in a two trait case, $\mu_{1}=\mu_{2}=0$ indicates that there is niche partitioning along both trait dimensions (Figure 1d), but $\mu_{1}=0$ and $\mu_{2} \neq 0$ indicate that there is niche partitioning along the first trait dimension and hierarchical competition along the second trait dimension (Figure 1e). Finally, $\rho_{21}$ determines if trait differences independently contribute to the pairwise interaction coefficients $\left(\rho_{21}=0\right.$; Figure 1d,e) or if they interact $\left(0<\left|\rho_{21}\right|<1\right.$, Figure 1f $)$.
Step 3-Integrating the abiotic and biotic filter

We assumed that species' covers could be modelled though a Lotka-Volterra competition model:

$$
\frac{1}{N_{i k}} \frac{d N_{i k}}{d t}=\frac{r_{i k}}{K_{i k}}\left(K_{i k}-\sum_{j} \alpha_{i j} N_{j k}\right),
$$

where $N_{i k}$ and $r_{i k}$ are, respectively, the percent cover and the intrinsic growth rate of species $i$ in site $k$. Note that this formulation of Lotka-Volterra competition model is slightly different from the formulation used in the twospecies coexistence framework of Chesson (2020, see supplementary information).

Within this model, the vector of all strictly positive species covers at equilibrium $N_{k}{ }^{*}$ satisfies the equation:

$$
N_{k}^{*}=\alpha^{-1} K_{k}
$$

where $\boldsymbol{K}_{\boldsymbol{k}}=\left[K_{i k}\right]$ is the vector of species carrying capacities, and $\boldsymbol{\alpha}=\left[\alpha_{i j}\right]$ is the matrix of pairwise interaction coefficients estimated as described above. As percent cover variables, $K_{i j}$ are less than or equal to 1 (see Equation 3) and interactions are strictly competitive, the estimated percent covers $N_{i k}$ are also less than or equal to 1 .

For a given set of parameters, we estimate the interaction matrix $\boldsymbol{\alpha}$ and computed its Moore-Penrose inverse $\boldsymbol{\alpha}^{-1}$. The Moore-Penrose inverse is identical to the usual matrix inverse when $\boldsymbol{\alpha}$ is invertible; it also approximates the inverse matrix when $\boldsymbol{\alpha}$ is not invertible, providing the least-squares solution when a single unique solution does not exist. We multiply $\alpha^{-1}$ to each site's vector of species carrying capacities (Equation 7). Species local cover estimated in this way can be negative, reflecting that this equilibrium state is not feasible. To find a feasible equilibrium, for each vector of equilibrium species covers, we sequentially set to 0 the species with the most negative cover and re-estimate the equilibrium state. This procedure is repeated until finding an equilibrium state where all remaining species covers are positive.

\section{A test of the framework}

We tested our framework on a data set from a complex ephemeral wetland in New Zealand (latitude $44.374143^{\circ} \mathrm{S}$, longitude $169.890052^{\circ} \mathrm{E}$ ). The herbaceous vegetation is dominated by grasses (e.g. Agrostis capillaris, Anthoxanthum odoratum), sedges (of genera Carex and Eleocharis) and forbs (e.g. Lilaeopsis ruthiana, Lobelia perpusilla). In wetland ecosystems, plant community structure is filtered by flooding duration (Moor et al., 2017) but is also determined by plant competition (Merlin et al., 2015).

In this test, we assumed that plant community assembly is determined by three functional traits (root 
porosity, height and SLA) that are filtered by flooding duration (abiotic filter), and by plant-plant competition (biotic filter).

Analyses of the data set are available in previous studies (Purcell et al., 2019; Tanentzap \& Lee, 2017; Tanentzap et al., 2014). Detailed methods about data collection are available in the supplementary materials. We analysed the vegetation structure with a subset of the complete data set (see Supplementary materials): 67 quadrats $25 \times 25 \mathrm{~cm}$ in size set along four transects that run from the lowest point of the basin and advance upslope to the kettle hole margin. Foliar cover was estimated for each species using the following cover estimates: $0 \%, 0.5 \%, 1 \%, 2 \%, 3 \%, 4 \%, 5 \%, 10 \%, 15 \%, 20 \%$, $30 \%, 40 \%, \ldots, 100 \%$. We restricted the analysis to the 15 most abundant species in the study area for which we sampled traits on at least 20 individuals. These species collectively represented at least $80 \%$ of the total of plant cover in each quadrat (Pakeman \& Quested, 2007). In each quadrat, a single individual of each species present in the quadrat was collected. Vegetative height, specific leaf area (SLA) and root porosity were measured on each collected individual (see supplementary information).

To estimate the carrying capacities with Traitspace, root porosity, as a percentage variable, was logittransformed. Height and SLA trait values were logtransformed prior to the analysis to approach a normal distribution. We modelled the relationship between root porosity, SLA, height and the flooding gradient and weighted trait observations by species cover. The intraspecific trait distribution of each species was modelled using a multivariate normal distribution (R-function mclust::dens Scrucca et al., 2016). We then modelled the probability of occurrence of each species in each site given the local duration of flooding using the Traitspace framework described earlier.

To calibrate the interaction matrices, we used the logarithm of species maximum height along the gradient (calculated as the $95 \%$ quantile of each species' height values) and the logarithm of species median SLA and species median root porosity.

Using the Banquo framework, we tested a total of 18 assembly models. Combining the different steps described above, those assembly models aim to solve for each site $k$ the following equation to estimate the vector of species cover $N_{k}^{*}$ at equilibrium:

$$
\left[\begin{array}{l}
\left.a\left(\frac{P\left(S_{1 k} \mid E_{k}\right)}{\max _{i, k}\left(P\left(S_{i k} \mid E_{k}\right)\right)}\right)^{b}, a\left(\frac{P\left(S_{2 k} \mid E_{k}\right)}{\max _{i, k}\left(P\left(S_{i k} \mid E_{k}\right)\right)}\right)^{b}, \cdots\right] \\
-\alpha N_{k}^{*}=0 .
\end{array}\right.
$$

(Model 1) One null model without any assembly processes: Species carrying capacities are equal in every site $(a \in[0,1], b=0)$ and there is no interspecific competition $(\alpha=I)$.
(Model 2) One abiotic filtering model: Species carrying capacities are estimated by the Traitspace framework (Equation 3, $a \in[0,1], b>0$ ) and there is no interspecific competition $(\boldsymbol{\alpha}=\boldsymbol{I})$.

(Models 3-10) Eight biotic filtering models without abiotic filtering: Species carrying capacities are equal in every site (Equation 3, $a \in[0,1], b=0$ ), but species cover is determined by competitive interactions $(\boldsymbol{\alpha} \neq \boldsymbol{I})$ that could be constant $(\sigma \rightarrow \infty$, Model 3), depend on a single trait (Models 4-6), depend on a combination of two traits (Models 7-9) or depend on all three traits (Model 10).

(Models 11-18) Eight abiotic and biotic filtering models: Species carrying capacities are estimated by the Traitspace framework (Equation 3, $a \in[0,1], b>0$ ) and species cover is also impacted by competitive interactions $(\boldsymbol{\alpha} \neq \boldsymbol{I})$ that could be constant $(\sigma \rightarrow \infty$, Model 11), depend on a single trait (Models 12-14), depend on a combination of two traits (Models 15-17) or depend on all three traits (Model 18).

We summarise the characteristics of each assembly model and their parameters in Table 1.

\section{Calibration and comparison}

We used the likelihood function proposed by Irvine et al. (2019) given that observed species covers were recorded as percent cover classes. Briefly, this likelihood function links the ordinal observations of plant cover to a latent beta distribution of mean $N_{i k}{ }^{*}$ (in our case estimated by the assembly models) and uncertainty parameter $\varphi$. One drawback of using the beta distribution is that it cannot model zero percent covers. To circumvent that issue, we added a small offset $(0.05 \%)$ to zero percent cover values, as suggested by Irvine et al. (2019).

Depending on the assembly models, there were two (null model) to 13 parameters (abiotic + biotic model with height, root porosity and SLA) to estimate. We set regularising priors on all parameters (Banner et al., 2020): we avoid making a priori assumptions about the nature of the relationship between traits, carrying capacities, pairwise interactions and species cover, but we limited the extent of the parameter space that was uninformative. Details about the prior functions and their hyperparameterisation are available in the supplementary materials.

We used a Differential Evolution Markov Chain Monte Carlo algorithm (DEzs MCMC, R-package BayesianTools, Hartig et al., 2017) to estimate the posterior distributions of the parameters. For each model, we ran four chains for $4 \times 10^{5}$ steps. We initialised the $Z$ matrix, that contains the current and past states of the MCMC chains, with four 1000-step runs of a differential evolution optimiser (Oberpriller et al., 2021; R-package DEoptim, Ardia et al., 2011). $Z$ is the matrix that contains the current and past states of the MCMC chains. By initialising it with the output of DEoptim, this 


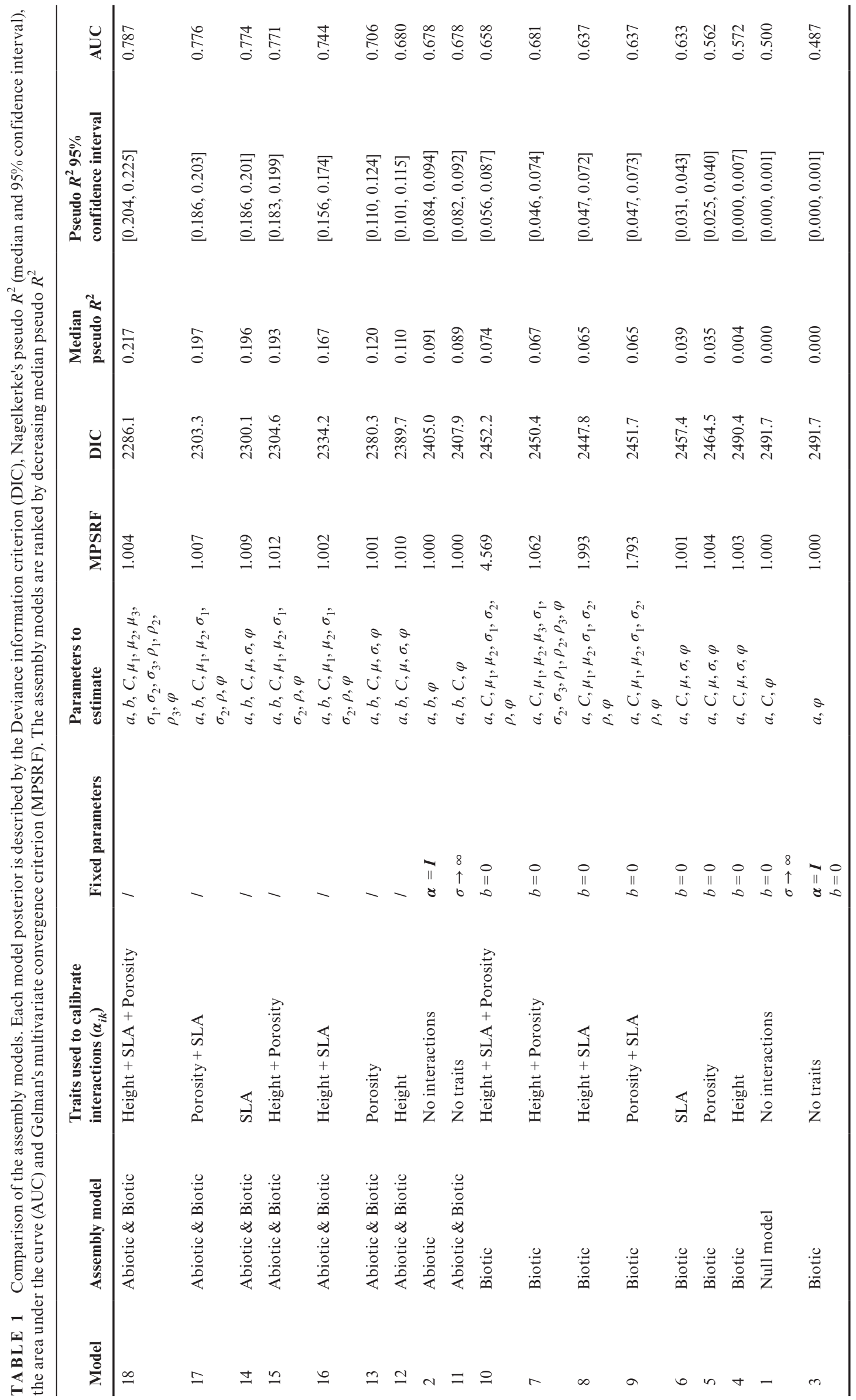


allows us to start the chains nearer to the optimum and thus avoids massive burn-in. Convergence was assessed through Gelman's multivariate convergence criterion (MPSRF, Gelman et al., 2014).

\section{Assembly model comparison}

We compared the fits of the calibrated models using two metrics: the Deviance information criterion (DIC, Gelman et al., 2014) and Nagelkerke's pseudo $R^{2}$ metric (Nagelkerke, 1991), which lends itself well to models that use a beta distribution and gives an indication of the variance they explain (Nakagawa \& Schielzeth, 2013). Nagelkerke's pseudo $R^{2}$ was calculated from the ratio of a model's posterior likelihood and the likelihood of the null model (see above). Furthermore, we evaluate the ability of the models to predict species presence/absence by evaluating the area under the curve (AUC) scores of each assembly model (R-package pROC Robin et al., 2011).

\section{Code availability}

The R-scripts and data to run the analysis are available at https://doi.org/10.5281/zenodo.5749620.

\section{RESULTS}

\section{Relationship between flooding and functional traits}

Root porosity increased significantly with flooding duration $\left(t=7.714, d f=224, p<0.0001\right.$. Adjusted $R^{2}=0.21$, Figure 2). Plant height decreased $(t=-3.91 ; d f=224$, $p=0.0001)$ and specific leaf area increased with flooding duration $(t=-3.10 ; d f=224, p=0.002)$, but these two latter relationships explained only a negligible portion of trait variation along the flooding duration gradient (Height adjusted $R^{2}=0.036$; SLA adjusted $R^{2}=0.060$ ).

\section{Model comparison}

Regardless of the performance statistic, there was a clear hierarchy across the assembly models (Table 1). The biotic models without abiotic filtering performed the worst (DIC $\geq 2443.2$, median pseudo $R^{2} \leq 0.074$, AUC $\leq 0.681$ ). Three among them did not converge (Gelman's multivariate convergence criterion over 1.1), but the associated pseudo $R^{2}$ was low across their posterior distribution (Table 1). The abiotic model without biotic interactions performed better than the biotic models (DIC: 2405 , median pseudo $R^{2}$ : 0.091, AUC: 0.678). The models that included both abiotic filtering and biotic interactions performed the best both in explained plant cover variance $\left(0.089 \leq\right.$ median pseudo $R^{2} \leq 0.217$; $2286.0 \leq \mathrm{DIC} \leq 2407.9)$ and species presence/absence $(0.678 \leq$ AUC $\leq 0.787)$. Among these models, the model that assumed fixed pairwise interaction coefficients among competing species had the lowest performance (median pseudo $R^{2}$ : 0.089; DIC: 2407.9 , $\mathrm{AUC}=0.678$ ), while the SLA-based interaction models were the best performing. Among these, the model that calibrated biotic interactions using all three functional traits was the best-fitting model (DIC: 2286.1, pseudo $R^{2}$ : 0.217, AUC: 0.787).

\section{Calibrated pairwise interaction matrices}

Among the assembly models that included both abiotic filtering and biotic interactions, the pairwise interaction matrix calibrated with all three traits was the best supported by the data. It indicated a situation with a mixture of niche partitioning and hierarchical competition across the three functional traits ( $\mu_{1}$ (root porosity): 95\% IQ [-0.067, 2.48]; $\mu_{2}$ (SLA): $95 \%$ IQ [-2.10, $0.05] ; \mu_{3}$ (height): $95 \%$ IQ [-2.81, 0.0048], Supplementary Figure 23). More specifically, the distributions of $\mu_{1}, \mu_{2}$ and $\mu_{3}$ indicated a competitive advantage for species that have high maximum height, large SLA values and small values of root porosity (Figure $3 \mathrm{e}, \mathrm{f}$ ).

\section{Comparison between the abiotic model and the abiotic and biotic assembly models}

We compared the abiotic model to the best assembly model (i.e. abiotic and biotic model with pairwise interactions calibrated all three functional traits). The abiotic model did not predict species absences well. The distribution of cover values was thus approximately normal around a median value of $3.30 \%$ (Supplementary Figure 5). Consequently, species presence along the flooding gradient was often overestimated with numerous species being predicted to be present in sites where they were not observed (e.g. see Epilobium angustum, Figure 4). In contrast, when biotic interactions are included, the assembly model tends to predict more absences and non-null percent cover values were less even among species (Figure 4, Supplementary Figure 5).

\section{DISCUSSION}

Predictive models of community assembly have focused on incorporating abiotic filters and have generally ignored biotic interactions. Here, we show that trait-based assembly rules can be used to directly model species abundances in communities by simultaneously accounting for both abiotic filtering and competitive interactions (Keddy, 1992). There are two major implications of this study. First, we introduced a trait-based formulation of pairwise competitive interactions that allowed us to calibrate 210 interaction coefficients from observational 


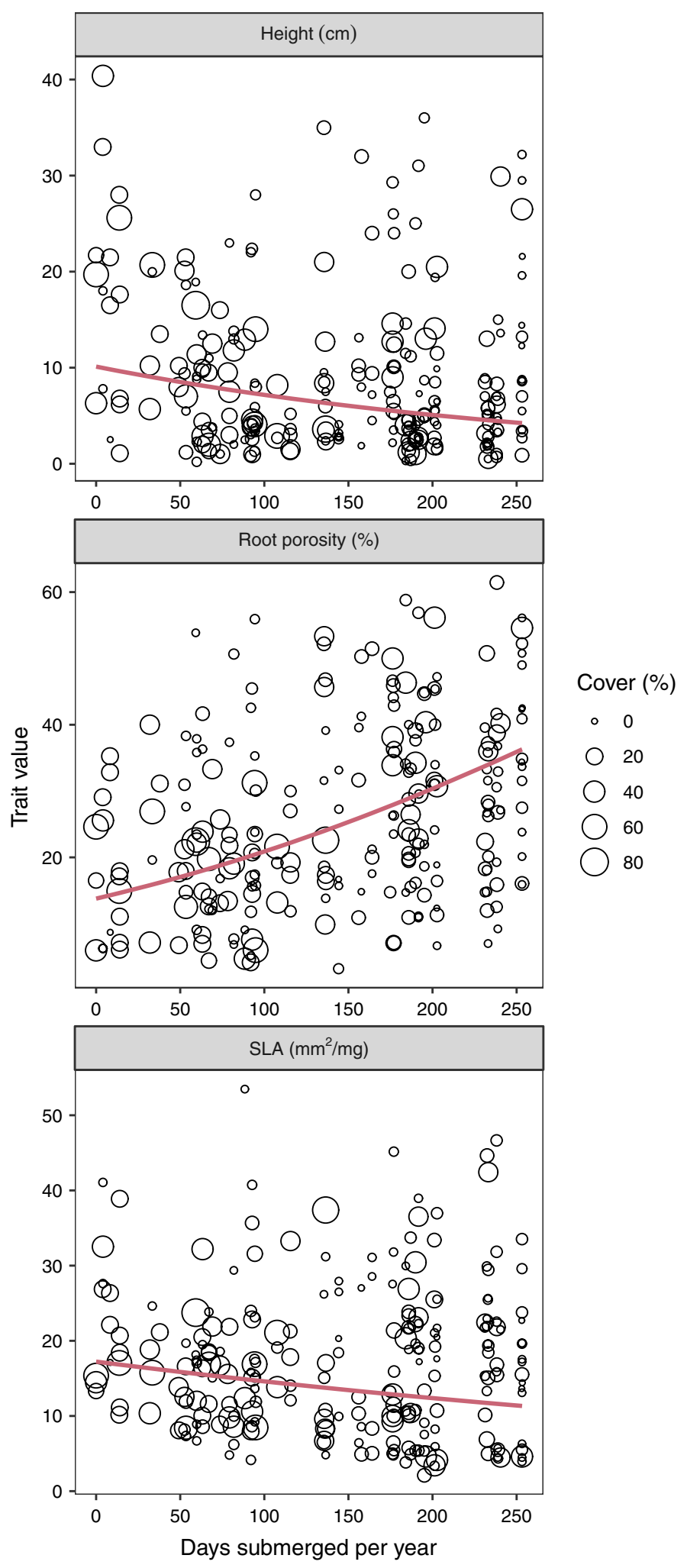

F I G U R E 2 Relationship between the duration of flooding and plant traits. From top to bottom: height, root porosity and specific leaf area. Data point size is proportional to plant cover. The line indicates the modelled relationship used in the Traitspace framework. The three linear models were all statistically significant (Root porosity, adjusted $R^{2}=0.206, p<0.0001$; specific leaf area, adjusted $R^{2}=0.037, p=0.0021$; vegetative height, adjusted $\left.R^{2}=0.060, p=0.0001\right)$

data with up to 10 parameters. This new approach substantially improves our ability to infer interaction matrices with little additional complexity (Cabral et al., 2017;
Chalmandrier et al., 2021). Second, the inclusion of competitive interactions among species improved predictions of local plant covers, which bolsters the argument that 
(a)

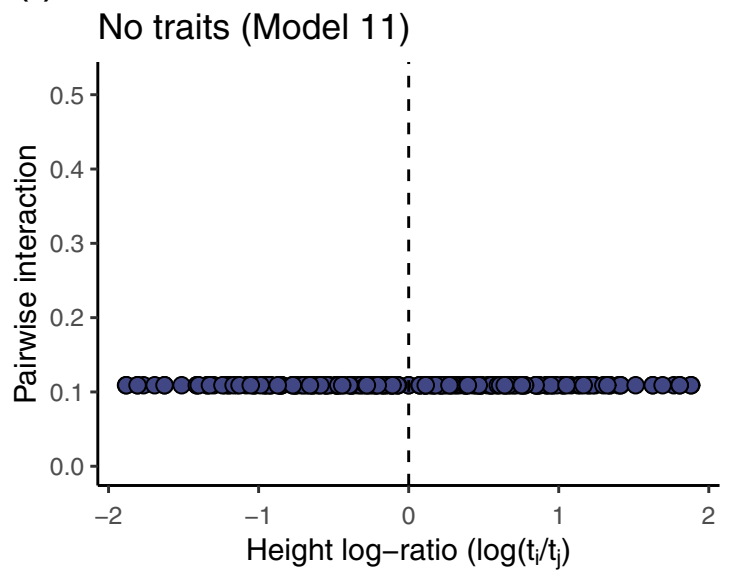

(c) Root porosity (Model 13)

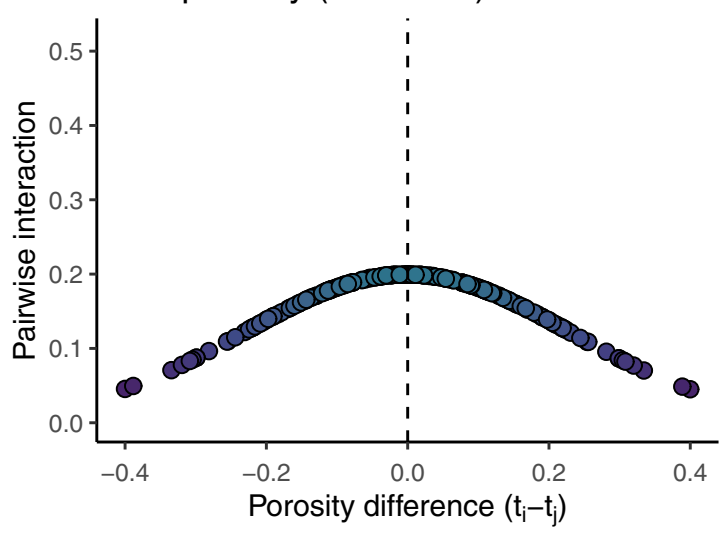

(e) Height, SLA \& Root porosity (Model 18)

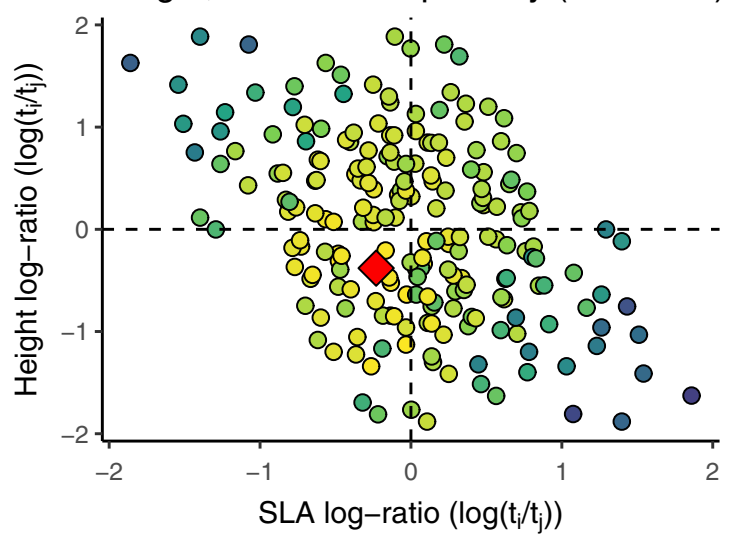

(b)

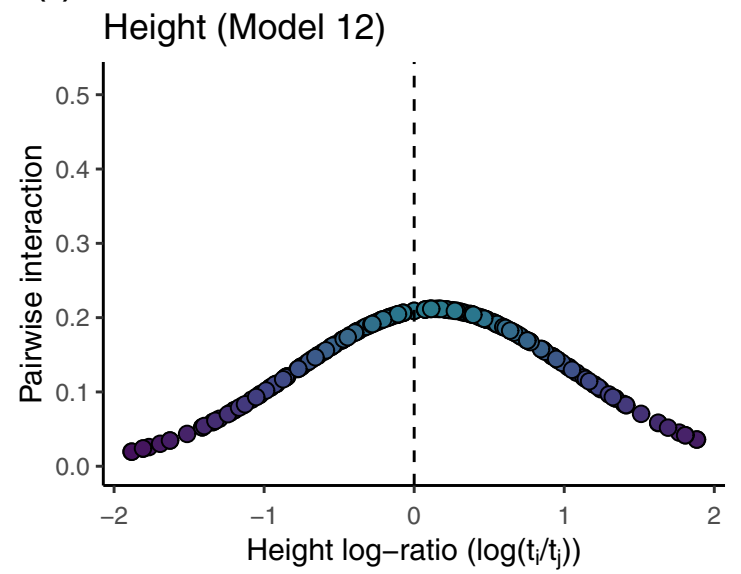

(d) SLA (Model 14)

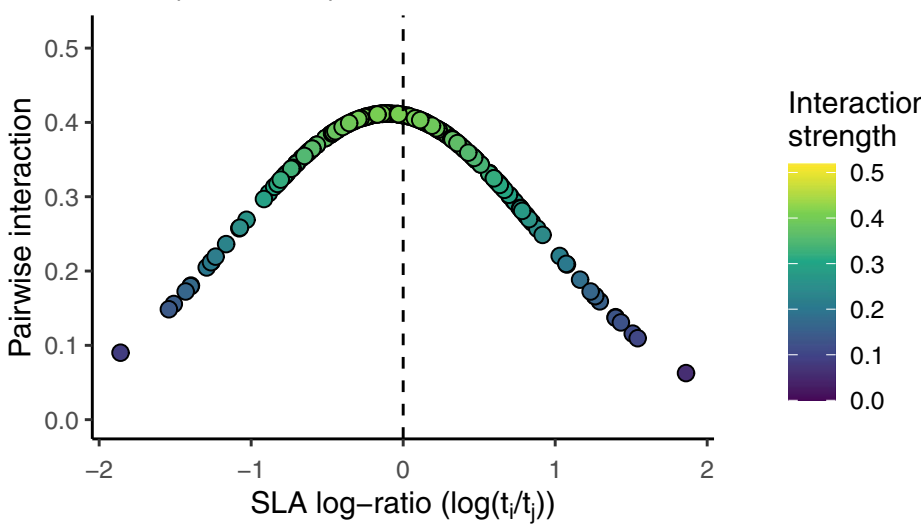

(f) Height, SLA \& Root porosity (Model 18)

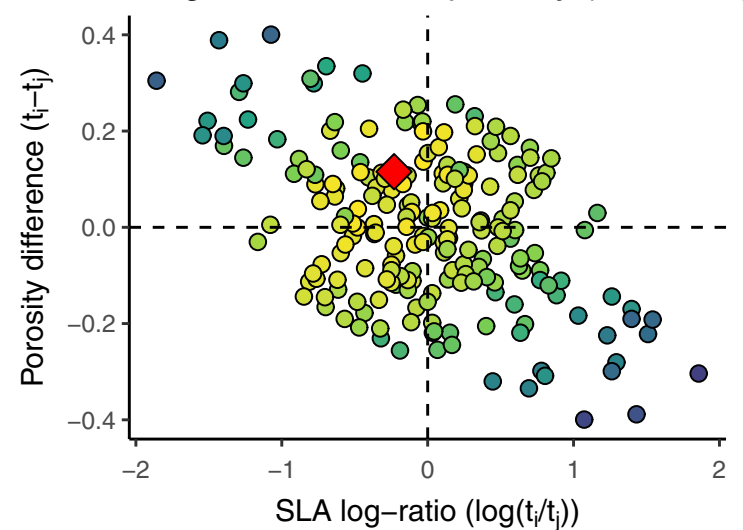

F IG U RE 3 Calibrated interspecific pairwise interaction coefficients of the abiotic and biotic assembly models at the median of parameter posterior distribution. (a) The competitive impact of species $j$ on species $i\left(\alpha_{i j}\right)$ is calibrated as a constant. (b-d) $\alpha_{i j}$ is calibrated as a function of a single trait difference (b: height; c: root porosity; d: SLA). (e, f) $\alpha_{i j}$ is calibrated as a function of all three trait differences (e: SLA vs. height differences; f: SLA vs. root porosity differences); to facilitate the interpretation of the two-trait plots (e, f), we indicated the position of the largest pairwise interaction coefficient value with an orange diamond

the modelling of species distribution must include both abiotic tolerances and species interactions (Alexander et al., 2015; Evans et al., 2016).

Our trait-based modelling approach explicitly specifies classical assembly mechanisms and evaluates their ability to predict species the local abundance using common modelling statistics. This contrasts with both diversity pattern analyses and neutral models (Hubbell, 2001; Webb et al., 2002) that test the structure of communities but do not provide an explicit modelling of each individual species. The core feature of the Banquo model is the formulation and calibration of pairwise competition coefficients 

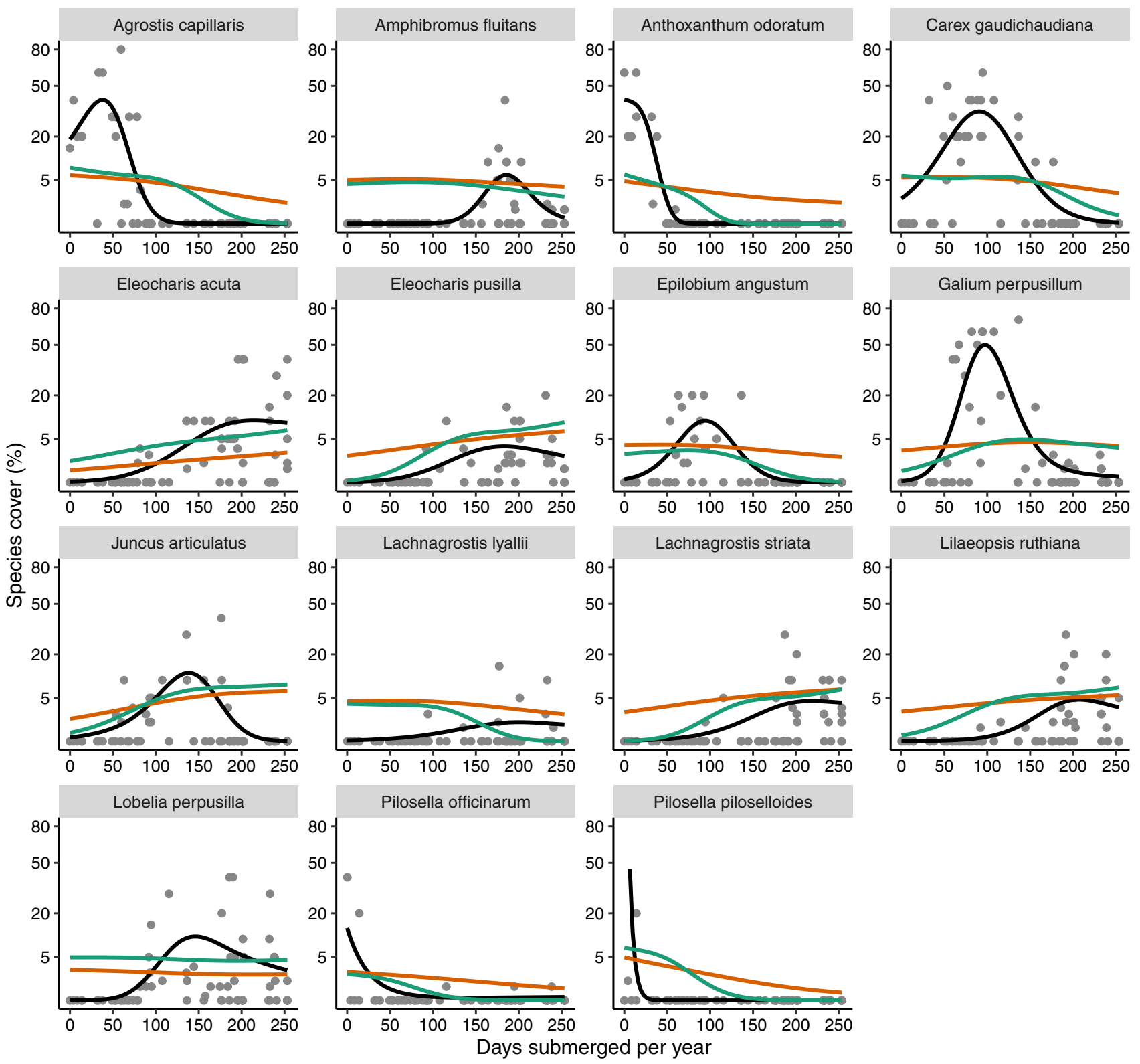

- Observed — Abiotic model — Abiotic + Biotic model

F I G U R E 4 Comparison of observed and modelled species cover along the flooding gradient. Orange response curves are fitted on the species cover as predicted by the abiotic model (Model 2, see Table 1). Green response curves are fitted on the species cover as predicted by the best abiotic and biotic model (Model 18, see Table 1). Both refer to species cover prediction at the median of parameter posterior distribution of their respective assembly model. Black nonlinear response curves are fitted directly to the observed cover for each species. All curves are fitted using a generalised additive model

as a function of species trait differences. Compared to estimating pairwise interaction coefficients individually, this considerably reduces the number of parameters to estimate (Chalmandrier et al., 2021; Zakharova et al., 2019). This formulation was directly inspired by coexistence theory and how it has informed the study of functional diversity patterns (Chesson, 2000; HilleRisLambers et al., 2012). Traditionally, diversity pattern studies have assumed that niche partitioning was the main competition mechanism behind community assembly (MacArthur \&
Levins, 1967). Niche partitioning would promote the coexistence of functionally dissimilar species and oppose itself to environmental filtering that promotes the coexistence of functionally similar species. In recent years, this framework has been criticised (Kraft, Adler, et al., 2015; Münkemüller et al., 2020) as coexistence theory posits that functionally similar species can coexist also through hierarchical competition, and more generally that coexistence is determined by a balance between niche partitioning and hierarchical competition (Adler et al., 2007). 
Our framework has the benefit of not assuming niche partitioning or hierarchical competition as the main trait-based competitive mechanism among species, but rather permits the fit of a mixture of the two processes. Our empirical example illustrates that the pairwise interaction matrices of the assembly models were consistent with strong niche partitioning among species but with a degree of hierarchical competition advantaging species with high stature and high SLA.

However, our approach is essentially phenomenological and does not explicitly model the mechanisms behind plant-plant competition. Future developments may aim at formulating competition as an explicit function of species' ability to consume local soil resources (Letten $\&$ Stouffer, 2019) or intercept light (Falster et al., 2017). Moreover, Falster et al. (2021) showed that pairwise interactions that arise from those models are not necessarily Gaussian functions of trait differences. However, implementing those more mechanistic frameworks would also require modelling species interactions as non-linear functions of species abundances (e.g. Letten \& Stouffer, 2019), thus going beyond the classic Lotka-Volterra competition model at the core of our framework. Beyond competition, a more complex modelling of plant biotic interactions could also include facilitative interactions or acknowledge that the nature of species interactions can shift along environmental gradients (Bimler et al., 2018; Maestre et al., 2009). Finally, phylogenetic differences may be used, albeit with caution, as a proxy for missing functional trait dimensions that are evolutionary conserved (Münkemüller et al., 2020; Webb et al., 2002).

One of the limitations of established trait-based models is that they tend to overestimate species occurrences (e.g. Merow et al., 2011). This drawback also affects other types of biodiversity models such as stacked species distribution models (Pottier et al., 2013) leading to inaccurate predictions of community structure at small spatial scales (Thuiller et al., 2015). This has been interpreted as a consequence of not properly accounting for biotic interactions. Our study supports for this conjecture: including competitive interactions improves the modelling of species occurrences and further decreases the predicted diversity ( $\alpha$-diversity) and increases the predicted turnover ( $\beta$-diversity) bringing them closer to the observed diversity values (Supplementary Figure 4). Our results follow the classical expectation that the realised niche of species is smaller than the fundamental niche because species interactions limit where species actually occur (MacArthur \& Levins, 1967). More specifically, the abiotic filtering model predicts remarkably even species abundances (Figure 4, Supplementary Figure 5), in contrast to the usual strong heterogeneity that characterises species abundance distributions (McGill et al., 2007). The inclusion of biotic interactions predicts a more realistic distribution of species abundances within communities with more species absences and a stronger hierarchy among species (Figure 4, Supplementary Figure 5).
By applying our framework, we were able to get insights into the ecological mechanisms that determine wetland community structure along a strong flooding gradient. First, we found that a trait-based model of abiotic filtering led to a modest improvement in model fit compared to the null model. This suggested flooding filtered the species pool primarily by porous root tissue that enhances the ability of species to tolerate flooded and anoxic soil (Moor et al., 2017; Tanentzap $\&$ Lee, 2017). When we added trait-based competitive interactions to this assembly model, we significantly improved the modelling of species covers and, consequently, of community structure. The 'best' model was the model that calibrated biotic interactions as a function of all three functional traits. Out of all single trait models, the SLA-based model explained the most species cover variance almost to the extent of the three functional traits model. This suggests that competitive interactions among plants in this ecosystem could be mainly mediated through leaf economics (Tanentzap \& Lee, 2017; Violle et al., 2009) and, to a lesser extent, stature and root morphology. Root porosity in itself is not usually considered to influence competitive interactions. However, it is related to root tissue density which aligns with the resource conservation axis within the root economics space (Bergmann et al., 2020; Freschet et al., 2021).

However, even the best assembly model explained a relatively modest portion of species abundances. This points both to the limitations of the available data and of our framework. Only root porosity was found to vary, and only moderately, along the flooding gradient (adjusted $R^{2}=0.21$ ). Thus, the modelled carrying capacities of species along the flooding did not vary as strongly as could be a priori expected (see Figure 4). The intraspecific variability of root porosity was substantial (32\% of total root porosity variance was intraspecific) and may dampen our ability to use this trait to model species' abiotic niches (Read et al., 2017). It is also possible that other unmeasured functional traits may be involved in the filtering of species along the flooding gradient (Moor et al., 2017).

\section{CONCLUSION}

It has been argued that complex ecological processes can be modelled with limited data input by leveraging the generality of functional traits (McGill et al., 2006). In community ecology, functional traits are mainly used in diversity pattern analyses codified by assembly theory (Keddy, 1992). Those analyses have numerous pitfalls: non-random functional diversity patterns can be interpreted in multiple ways thus rendering difficult a confident inference of community assembly rules (Kraft, Adler, et al., 2015; Münkemüller et al., 2020). In contrast, our approach specifies explicit assembly rules and 
model directly local species abundances. Ultimately, our framework provides a process-based approach to predict community structure and quantify its statistical support. Such trait-based modelling opens a new general way to model natural communities and will improve our ability to understand and predict biodiversity structure and dynamics under global change.

\section{ACKNOWLEDGEMENTS}

This research was supported by funding from both the University of Waikato and Manaaki Whenua-Landcare Research. John Payne of Landcare Research kindly provided the capacitance probe water level data. We call our new model, 'Banquo', in honour of Bill Shipley, who, in his foundational book (Shipley 2010), was the first to highlight Banquo's desire to predict 'which grain will grow and which will not' in Shakespeare's Macbeth. LC acknowledges a postdoctoral fellowship at the University of Wyoming and funding from the European Union's Horizon 2020 research and innovation programme under the Marie Skłodowska-Curie grant agreement No 840946 (Project 'CLIMB'). DBS acknowledges the support of a Rutherford Discovery Fellowship and the Marsden Fund Council from New Zealand Government funding, both of which are managed by the Royal Society of New Zealand Te Apārangi (RDF-13-UOC-003 and 16-UOC-008), and an Erskine Grant from the University of Canterbury. Open Access funding enabled and organized by Projekt DEAL.

\section{AUTHORSHIP}

LC, DBS and DCL led the study. LC developed and ran the analyses and wrote the first draft. ASTP, WGL, AJT and DCL collected the data. All authors contributed to the writing of the paper.

\section{PEER REVIEW}

The peer review history for this article is available at https://publons.com/publon/10.1111/ele.13980.

\section{DATA AVAILABILITY STATEMENT}

The R-scripts and data to run the analysis are currently available at the archived repository https://doi. org/10.5281/zenodo.5749620.

\section{ORCID}

Loüc Chalmandrier (10) https://orcid.org/0000-0002-2631-0432 Daniel B. Stouffer (1D https://orcid.org/0000-0001-9436-9674 William G. Lee (1D https://orcid.org/0000-0001-7717-0807 Andrew J. Tanentzap (10 https://orcid.org/0000-0002-2883-1901 Daniel C. Laughlin (10) https://orcid.org/0000-0002-9651-5732

\section{REFERENCES}

Adler, P.B., HilleRisLambers, J. \& Levine, J.M. (2007) A niche for neutrality. Ecology Letters, 10, 95-104.

Alexander, J.M., Diez, J.M. \& Levine, J.M. (2015) Novel competitors shape species' responses to climate change. Nature, 525, 515-518.
Ardia, D., Boudt, K., Carl, P., Mullen, K. \& Peterson, B.G. (2011) Differential evolution with DEoptim: an application to nonconvex portfolio optimization. The R Journal, 3, 27-34.

Banner, K.M., Irvine, K.M. \& Rodhouse, T. (2020) The use of Bayesian priors in ecology: the good, the bad, and the not great. Methods in Ecology and Evolution, 11(8), 882-889.

Bergmann, J., Weigelt, A., van der Plas, F., Laughlin, D.C., Kuyper, T.W., Guerrero-Ramirez, N. et al. (2020) The fungal collaboration gradient dominates the root economics space in plants. Science Advances, 6, eaba3756.

Bimler, M.D., Stouffer, D.B., Lai, H.R. \& Mayfield, M.M. (2018) Accurate predictions of coexistence in natural systems require the inclusion of facilitative interactions and environmental dependency. Journal of Ecology, 106, 1839-1852.

Cabral, J.S., Valente, L. \& Hartig, F. (2017) Mechanistic simulation models in macroecology and biogeography: state-of-art and prospects. Ecography, 40, 267-280.

Chalmandrier, L., Hartig, F., Laughlin, D.C., Lischke, H., Pichler, M., Stouffer, D.B. et al. (2021) Linking functional traits and demography to model species-rich communities. Nature Communications, 12, 2724.

Chalmandrier, L., Münkemüller, T., Colace, M.-P., Renaud, J., Aubert, S., Carlson, B.Z. et al. (2017) Spatial scale and intraspecific trait variability mediate assembly rules in alpine grasslands. Journal of Ecology, 105, 277-287.

Chesson, P. (2000) Mechanisms of maintenance of species diversity. Annual Review of Ecology and Systematics, 31(1), 343-366.

Chesson, P. (2020) Species coexistence. In: McCAnn, K.S. \& Gellner, G. (Eds.) Theoretical Ecology: concepts and applications. Oxford: Oxford University Press, pp. 5-27.

D’Amen, M., Dubuis, A., Fernandes, R.F., Pottier, J., Pellissier, L. \& Guisan, A. (2015) Using species richness and functional traits predictions to constrain assemblage predictions from stacked species distribution models. Journal of Biogeography, 42, 1255-1266.

Damgaard, C.F. \& Irvine, K.M. (2019) Using the beta distribution to analyse plant cover data. Journal of Ecology, 107, 2747-2759.

Evans, M.E., Merow, C., Record, S., McMahon, S.M. \& Enquist, B.J. (2016) Towards process-based range modeling of many species. Trends in Ecology \& Evolution, 31, 860-871.

Falster, D.S., Brännström, Å., Westoby, M. \& Dieckmann, U. (2017) Multitrait successional forest dynamics enable diverse competitive coexistence. Proceedings of the National Academy of Sciences of the United States of America, 114, E2719-E2728.

Falster, D.S., Kunstler, G., FitzJohn, R.G. \& Westoby, M. (2021) Emergent shapes of trait-based competition functions from resource-based models: a Gaussian is not normal in plant communities. The American Naturalist, 198, 253-267.

Forrester, P.J. \& Zhang, J. (2020) Parametrising correlation matrices. Journal of Multivariate Analysis, 178, 104619.

Freschet, G.T., Roumet, C., Comas, L.H., Weemstra, M., Bengough, A.G., Rewald, B. et al. (2021) Root traits as drivers of plant and ecosystem functioning: current understanding, pitfalls and future research needs. New Phytologist, 232, 1123-1158.

Gelman, A., Hwang, J. \& Vehtari, A. (2014) Understanding predictive information criteria for Bayesian models. Statistics and Computing, 24, 997-1016.

Hartig, F., Minunno, F. \& Paul, S. (2017) BayesianTools: generalpurpose MCMC and SMC samplers and tools for Bayesian statistics. R package.

HilleRisLambers, J., Adler, P.B., Harpole, W.S., Levine, J.M. \& Mayfield, M.M. (2012) Rethinking community assembly through the lens of coexistence theory. Annual Review of Ecology, Evolution, and Systematics, 43, 227.

Hubbell, S.P. (2001) The unified neutral theory of biodiversity and biogeography. Princeton, New Jersey, USA: Princeton University Press.

Irvine, K.M., Wright, W.J., Shanahan, E.K. \& Rodhouse, T.J. (2019) Cohesive framework for modelling plant cover class data. Methods in Ecology and Evolution, 10, 1749-1760. 
Keddy, P.A. (1992) Assembly and response rules: two goals for predictive community ecology. Journal of Vegetation Science, 3, 157-164.

Kraft, N.J.B., Adler, P.B., Godoy, O., James, E.C., Fuller, S. \& Levine, J.M. (2015) Community assembly, coexistence and the environmental filtering metaphor. Functional Ecology, 29, 592-599.

Kraft, N.J., Godoy, O. \& Levine, J.M. (2015) Plant functional traits and the multidimensional nature of species coexistence. Proceedings of the National Academy of Sciences of the United States of America, 112, 797-802.

Laughlin, D.C., Joshi, C., van Bodegom, P.M., Bastow, Z.A. \& Fulé, P.Z. (2012) A predictive model of community assembly that incorporates intraspecific trait variation. Ecology Letters, 15, 1291-1299.

Letten, A.D. \& Stouffer, D.B. (2019) The mechanistic basis for higherorder interactions and non-additivity in competitive communities. Ecology Letters, 22, 423-436.

Lotka, A.J. (1926) Elements of physical biology. Baltimore: Williams \& Wilkins Co.

MacArthur, R. \& Levins, R. (1967) The limiting similarity, convergence, and divergence of coexisting species. The American Naturalist, 101(921), 377-385.

Maestre, F.T., Callaway, R.M., Valladares, F. \& Lortie, C.J. (2009) Refining the stress-gradient hypothesis for competition and facilitation in plant communities. Journal of Ecology, 97, 199-205.

McGill, B.J., Enquist, B.J., Weiher, E. \& Westoby, M. (2006) Rebuilding community ecology from functional traits. Trends in Ecology \& Evolution, 21, 178-185.

McGill, B.J., Etienne, R.S., Gray, J.S., Alonso, D., Anderson, M.J., Benecha, H.K. et al. (2007) Species abundance distributions: moving beyond single prediction theories to integration within an ecological framework. Ecology Letters, 10, 995-1015.

Merlin, A., Bonis, A., Damgaard, C.F. \& Mesléard, F. (2015) Competition is a strong driving factor in wetlands, peaking during drying out periods. PLoS One, 10, e0130152.

Merow, C., Latimer, A.M. \& Silander, J.A. (2011) Can entropy maximization use functional traits to explain species abundances? A comprehensive evaluation. Ecology, 92, 1523-1537.

Moor, H., Rydin, H., Hylander, K., Nilsson, M.B., Lindborg, R. \& Norberg, J. (2017) Towards a trait-based ecology of wetland vegetation. Journal of Ecology, 105, 1623-1635.

Münkemüller, T., Gallien, L., Pollock, L.J., Barros, C., Carboni, M., Chalmandrier, L. et al. (2020) Do's and don'ts when inferring assembly rules from diversity patterns. Global Ecology and Biogeography, 29, 1212-1229.

Nagelkerke, N.J. (1991) A note on a general definition of the coefficient of determination. Biometrika, 78, 691-692.

Nakagawa, S. \& Schielzeth, H. (2013) A general and simple method for obtaining $R^{2}$ from generalized linear mixed-effects models. Methods in Ecology and Evolution, 4, 133-142.

Narwani, A., Alexandrou, M.A., Oakley, T.H., Carroll, I.T. \& Cardinale, B.J. (2013) Experimental evidence that evolutionary relatedness does not affect the ecological mechanisms of coexistence in freshwater green algae. Ecology Letters, 16, 1373-1381.

Oberpriller, J., Cameron, D.R., Dietze, M.C. \& Hartig, F. (2021) Towards robust statistical inference for complex computer models. Ecology Letters, 24, 1251-1261.

Ovaskainen, O., Tikhonov, G., Norberg, A., Guillaume Blanchet, F., Duan, L., Dunson, D. et al. (2017) How to make more out of community data? A conceptual framework and its implementation as models and software. Ecology Letters, 20, 561-576.

Pakeman, R.J. \& Quested, H.M. (2007) Sampling plant functional traits: what proportion of the species need to be measured? Applied Vegetation Science, 10, 91-96.
Pottier, J., Dubuis, A., Pellissier, L., Maiorano, L., Rossier, L., Randin, C.F. et al. (2013) The accuracy of plant assemblage prediction from species distribution models varies along environmental gradients. Global Ecology and Biogeography, 22, 52-63.

Purcell, A.S.T., Lee, W.G., Tanentzap, A.J. \& Laughlin, D.C. (2019) Fine root traits are correlated with flooding duration while aboveground traits are related to grazing in an ephemeral wetland. Wetlands, 39, 291-302.

Read, Q.D., Henning, J.A. \& Sanders, N.J. (2017) Intraspecific variation in traits reduces ability of trait-based models to predict community structure. Journal of Vegetation Science, 28, $1070-1081$

Robin, X., Turck, N., Hainard, A., Tiberti, N., Lisacek, F., Sanchez, J.-C. et al. (2011) pROC: an open-source package for R and S+ to analyze and compare ROC curves. BMC Bioinformatics, 12, 77.

Scrucca, L., Fop, M., Murphy, T.B. \& Raftery, A.E. (2016) mclust 5: clustering, classification and density estimation using Gaussian finite mixture models. The R Journal, 8, 289.

Shipley, B. (2010) From plant traits to vegetation structure: chance and selection in the assembly of ecological communities. Cambridge: Cambridge University Press.

Spasojevic, M.J., Copeland, S. \& Suding, K.N. (2014) Using functional diversity patterns to explore metacommunity dynamics: a framework for understanding local and regional influences on community structure. Ecography, 37, 939-949.

Tanentzap, A.J. \& Lee, W.G. (2017) Evolutionary conservatism explains increasing relatedness of plant communities along a flooding gradient. New Phytologist, 213, 634-644.

Tanentzap, A.J., Lee, W.G., Monks, A., Ladley, K., Johnson, P.N., Rogers, G.M. et al. (2014) Identifying pathways for managing multiple disturbances to limit plant invasions. Journal of Applied Ecology, 51, 1015-1023.

Thuiller, W., Pollock, L.J., Gueguen, M. \& Münkemüller, T. (2015) From species distributions to meta-communities. Ecology Letters, 18, 1321-1328.

Violle, C., Garnier, E., Lecoeur, J., Roumet, C., Podeur, C., Blanchard, A. et al. (2009) Competition, traits and resource depletion in plant communities. Oecologia, 160, 747-755.

Violle, C., Navas, M.-L., Vile, D., Kazakou, E., Fortunel, C., Hummel, I. et al. (2007) Let the concept of trait be functional! Oikos, 116, 882-892.

Webb, C.O., Ackerly, D.D., McPeek, M.A. \& Donoghue, M.J. (2002) Phylogenies and community ecology. Annual Review of Ecology and Systematics, 33, 475-505.

Zakharova, L., Meyer, K.M. \& Seifan, M. (2019) Trait-based modelling in ecology: a review of two decades of research. Ecological Modelling, 407, 108703.

\section{SUPPORTING INFORMATION}

Additional supporting information may be found in the online version of the article at the publisher's website.

How to cite this article: Chalmandrier, L., Stouffer, D.B., Purcell, A.S.T., Lee, W.G., Tanentzap, A.J. \& Laughlin, D.C. (2022) Predictions of biodiversity are improved by integrating trait-based competition with abiotic filtering. Ecology Letters, 00, 1-13. Available from: https://doi.org/10.1111/ele.13980 